\title{
ELECTRON-POSITRON COLLISIONS AT 209 GEV IN LEP
}

\author{
G. Arduini, R. Assmann, R. Bailey, A. Butterworth, P. Collier, K. Cornelis, S. Fartoukh, \\ M. Lamont, G. Morpurgo, G. Roy, J. Wenninger, T. Wijnands, CERN, Geneva, Switzerland
}

\section{Abstract}

The Large Electron-Positron Collider (LEP) at CERN closed down in November 2000. During the year 2000 electron-positron collisions were established at centre-ofmass energies of up to $209 \mathrm{GeV}$. The energy reach of LEP was thus extended by another $7 \mathrm{GeV}$ compared to the year 1999. At the same time the luminosity production was kept high and the total delivered luminosity for the year was $233 \mathrm{pb}^{-1}$. High beam energy and high luminosity extended the discovery reach of LEP. The successful energy increase of LEP is analysed and the operation and performance in the regime of ultra-strong damping is described.

\section{INTRODUCTION}

The LEP discovery reach for the Standard Model Higgs boson was pushed from $95 \mathrm{GeV} / \mathrm{c}^{2}$ in 1998 to around $113 \mathrm{GeV} / \mathrm{c}^{2}$ in 2000 [1]. This increase was the result both of the increase of beam energy and the higher than expected luminosity production at these higher beam energies. How this was achieved are analysed herein.

Table 1 summarises the maximum beam energies, integrated luminosities and the average rate of luminosity production for 1994 to 2000 . The LEP performance was improved significantly over the years. From the total of $1000 \mathrm{pb}^{-1}$ per experiment delivered between 1989 and 2000 almost $70 \%$ were delivered in the last three years.

The Higgs discovery reach of LEP was maximised by a trade-off between luminosity and energy, some luminosity being sacrificed in the interest of production at the highest possible energies. This is discussed in detail in [1]. It is seen from Table 1 that the peak performance of 1999 was not reached during 2000. This reduction reflects the trade-off between maximising beam energy and integrated luminosity.

\begin{tabular}{|c|c|c|c|}
\hline Year & $\begin{array}{c}\text { Beam energy } \\
{[\mathbf{G e V}]}\end{array}$ & $\begin{array}{c}\text { Total } \\
\text { luminosity } \\
{\left[\mathbf{p b}^{-1}\right]}\end{array}$ & $\begin{array}{c}\text { Luminosity } \\
\text { rate } \\
{\left[\mathbf{p b}^{-1} / \mathbf{d a y}\right]}\end{array}$ \\
\hline 1994 & 45.6 & 64 & 0.31 \\
\hline 1995 & $45.6-70.0$ & 47 & 0.23 \\
\hline 1996 & $80.5-86.0$ & 25 & 0.17 \\
\hline 1997 & $91.0-92.0$ & 75 & 0.66 \\
\hline 1998 & 94.5 & 200 & 1.16 \\
\hline 1999 & $96.0-101.0$ & 254 & 1.35 \\
\hline 2000 & $100.0-104.5$ & 233 & 1.10 \\
\hline
\end{tabular}

Table 1: Overview of LEP performance 1994-2000.

\section{MAXIMUM BEAM ENERGY}

The maximum operational energy of LEP depended on a number of different parameters [2,3]:

Available accelerating RF voltage Installation of LEP2 superconducting cavities ended in 1999. There was therefore very little additional RF hardware at the start of the 2000 run. The main gain came from raising the accelerating gradient of the super-conducting RF cavities well above the design value of $6 \mathrm{MV} / \mathrm{m}[4]$.

Rate of RF trips The RF system was protected with about 10,000 interlocks. Interlocks can trip one klystron ( $100 \mathrm{MV}), 2$ klystrons, or dump the beam. Trips occurred on a statistical basis and were mainly produced by field emission, causing Helium level or pressure problems. Recovery was fast $(\sim 2 \mathrm{~min})$. The trip rate determined the required operating $\mathrm{RF}$ voltage margin: by working at an energy lower than the maximum possible it was possible to have RF trips and keep the beam [4]. The RF stability was improved with fast GPS based diagnostics, active damping of field oscillations and various hardware improvements.

Maximum horizontal beam size The horizontal beam size $\sigma_{\mathrm{x}}$ is proportional to beam energy $E$, the RMS horizontal dispersion $\mathrm{D}_{\mathrm{x}}^{\mathrm{rms}}$, the betatron function $\beta_{\mathrm{x}}$ and the horizontal damping partition number $\mathrm{J}_{\mathrm{x}}$ :

$$
\sigma_{x} \propto \sqrt{\beta_{x} / J_{x}} \cdot D_{x}^{r m s} \cdot E
$$

The increase of horizontal beam size with energy results in lower luminosity and larger background in the experiments. This compensated for by the use of a high $Q_{x}$ optics [5] and an increase of $J_{x}$ through an increase of the RF frequency. Up to $91.5 \mathrm{GeV}$ a $90^{\circ} / 60^{\circ}$ optics was used, above $91.5 \mathrm{GeV}$ a $102^{\circ} / 90^{\circ}$ optics took over. The tactic of increased $J_{x}$ reduces both beam energy and increases the RF voltage required (larger energy spread). For maximum beam energy, however, one should run with the lowest $\mathrm{J}_{\mathrm{x}}$ possible, and indeed, in 2000 increased horizontal beam size was accepted in the interests of increasing the beam energy.

Average bending radius The energy loss per turn is a function of beam energy $E$ and average bending radius $\rho$. The average bending radius can be changed operationally by using the horizontal dipole correctors [6].

The LEP energy was maximised in 2000 by optimising all of the above contributions. Due to the cost in energy of the RF voltage margin (200 MV corresponded to $\sim 1.6 \mathrm{GeV})$ a special ramping strategy was implemented. A physics fill was started at an energy equivalent to a 2 klystrons margin, after a while the beam was then 
ramped, under physics conditions, to an energy equivalent to a 1 klystron margin. Towards the end of a fill the beam energy was ramped to the maximum (no RF margin), the end of fill being given by the next RF trip. The contributions to the energy gain of $3.4 \mathrm{GeV}$ in 2000 with respect to 1999 is shown in Table 2. Luminosity production throughout 2000 at different energies is illustrated in Figure 1.

\begin{tabular}{|l|r|}
\hline Contribution & Energy gain \\
\hline Additional RF cavities & $0.14 \mathrm{GeV}$ \\
\hline Higher RF gradient & $0.96 \mathrm{GeV}$ \\
\hline Less RF margin & $1.60 \mathrm{GeV}$ \\
\hline Reduced RF frequency & $0.70 \mathrm{GeV}$ \\
\hline Increased bending radius & $0.17 \mathrm{GeV}$ \\
\hline Total & $\mathbf{3 . 5 3} \mathrm{GeV}$ \\
\hline
\end{tabular}

Table 2: Contributions to the energy increase in 2000.

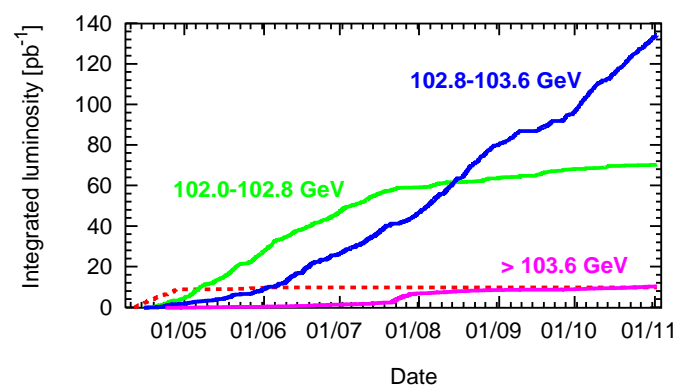

Figure 1: Luminosity production in 2000 . The three ranges correspond to 2, 1 and 0 klystrons margin (right hand numbers, from top to bottom).

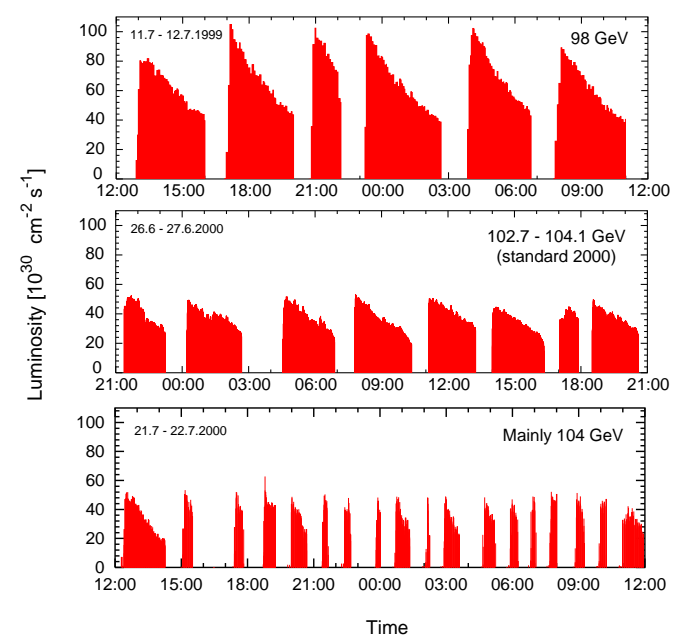

Figure 3: Instantaneous luminosity in LEP for three different days in 1999 and 2000. The different modes of operation for $98 \mathrm{GeV}, 102.7$ to $104.1 \mathrm{GeV}$ (standard running in 2000) and mainly $104 \mathrm{GeV}$ are clearly visible.

\section{LUMINOSITY PERFORMANCE}

Luminosity production was highest in 1999, as can be seen from Table 1 . Figure 2 shows the peak luminosity versus energy for the physics fills in 1998, 1999, and
2000. The beam-beam limit was not reached for beam energies above $70 \mathrm{GeV}$. The peak luminosities came down when the beam energy was raised to $100 \mathrm{GeV}$ and $101 \mathrm{GeV}$. The decrease of luminosity continued with the higher beam energies in 2000 . The reduction is mainly due to lower beam currents, shorter fills and larger horizontal beam sizes (see discussion above). Three different modes of LEP running are illustrated in Figure 4: $98 \mathrm{GeV}$ with a large RF voltage overhead (horizontal beam size reduced aggressively with $\mathrm{J}_{\mathrm{x}}=1.7$ ), mini-ramp strategy in 2000 , and dedicated running at $104 \mathrm{GeV}$ without any overhead.

\begin{tabular}{|c|c|c|c|c|c|}
\hline $\begin{array}{c}\text { Energy } \\
{[\mathbf{G e V}]}\end{array}$ & $\begin{array}{c}\boldsymbol{\xi}_{\mathbf{y}} \mathbf{p e r} \\
\mathbf{I P}\end{array}$ & $\begin{array}{c}\boldsymbol{\beta}_{\mathbf{x}}{ }^{*} / \boldsymbol{\beta}_{\mathbf{y}}{ }^{*} \\
{[\mathbf{m}]}\end{array}$ & $\begin{array}{c}\mathbf{i}_{\mathbf{b}} \\
{[\boldsymbol{\mu A}]}\end{array}$ & $\mathbf{J}_{\mathbf{x}}$ & $\begin{array}{c}\boldsymbol{\tau}_{\text {transv }} \\
{\left[\mathbf{T}_{\mathbf{0}}\right]}\end{array}$ \\
\hline 45.6 & 0.045 & $2.00 / 0.05$ & 320 & 1.0 & 721 \\
\hline 65.0 & 0.050 & $2.00 / 0.05$ & 400 & 1.0 & 249 \\
\hline 91.5 & 0.055 & $1.50 / 0.05$ & 650 & 1.6 & 89 \\
\hline 94.5 & 0.075 & $1.25 / 0.05$ & 750 & 1.8 & 81 \\
\hline 98.0 & 0.083 & $1.50 / 0.05$ & 800 & 1.6 & 73 \\
\hline 101.0 & 0.073 & $1.50 / 0.05$ & 700 & 1.3 & 66 \\
\hline$\geq 102.7$ & 0.055 & $1.50 / 0.05$ & 650 & 1.1 & $\leq 63$ \\
\hline
\end{tabular}

Table 3: Maximum vertical beam-beam parameter $\xi_{\mathrm{y}}$, IP beta functions $\beta_{\mathrm{x}}{ }^{*} / \beta_{\mathrm{y}}{ }^{*}$, bunch current $\mathrm{i}_{\mathrm{b}}$, horizontal damping partition number $\mathrm{J}_{\mathrm{x}}$, and transverse damping time $\tau_{\text {transv }}$ (in number of turns) for different beam energies.

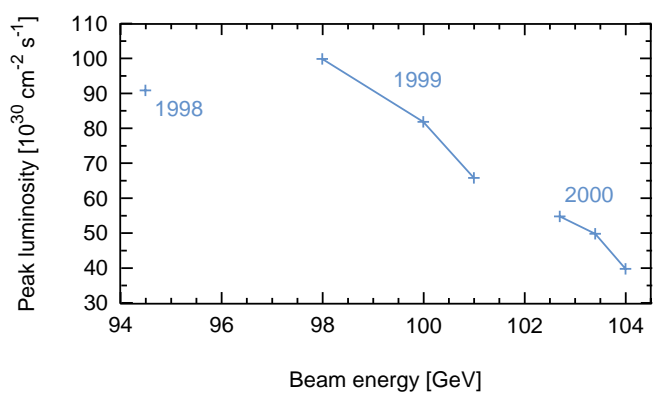

Figure 2: Peak luminosity in all physics fills between 1998 and 2000.

Peak luminosities reached $10^{32} \mathrm{~cm}^{-2} \mathrm{~s}^{-1}$ and the vertical beam-beam parameter 0.083 per IP at $98 \mathrm{GeV}$. A higher beam-beam limit at high energy is the result of the strong transverse damping (60 turns at $104.4 \mathrm{GeV}$ compared to 721 turns at $45.6 \mathrm{GeV}$ - see Table 3). Figure 4 shows the vertical beam-beam parameter for a $98 \mathrm{GeV}$ fill versus bunch current. The data is compared to the expected behaviour without beam-beam blow-up and a fit [10]. From the fit a beam-beam limit can be inferred at $\xi_{\mathrm{y}}=0.115$ and an unperturbed vertical emittance of $0.1 \mathrm{~nm}$. Though there was an emittance blow-up of up to $\sim 70 \%$, LEP did not reach the beam-beam limit at high energies.

The strong transverse damping allowed a jump of the third integer resonance for a high $\mathrm{Q}_{\mathrm{x}}$ working point of 0.36 with both better luminosity and backgrounds.

The release from the beam-beam limit allowed thorough optimisation of the vertical beam size and consider- 
able reduction was achieved by targeting coupling and residual vertical dispersion.

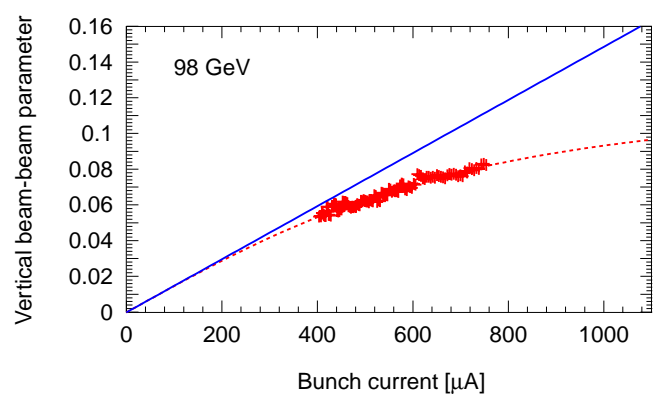

Figure 4: Vertical beam-beam parameter versus bunch current. The data is compared to the not beam-beam limited case and a fit [10].

The integrated luminosity was optimised by both maximising the instantaneous luminosity and the time available for physics. As shown in Table 4, the overhead due to the magnet cycling, injection at $22 \mathrm{GeV}$, ramping to high energy and setting up for physics was reduced from $110 \mathrm{~min}$ in 1998 to $65 \mathrm{~min}$ in 2000 . Higher beam intensities from the injector chain, increased injection efficiency, and double ramp speed contributed to this important improvement. In addition, an automated control of the horizontal damping partition number $\mathrm{J}_{\mathrm{x}}$ (as a function of the available RF voltage) was implemented.

\begin{tabular}{|c|c|}
\hline Year & Overhead per fill \\
\hline 1998 & $110 \mathrm{~min}$ \\
\hline 1999 & $93 \mathrm{~min}$ \\
\hline 2000 & $65 \mathrm{~min}$ \\
\hline
\end{tabular}

Table 4: Average overhead per physics fill.

\section{CONCLUSIONS}

The Large Electron-Positron Collider (LEP) at CERN completed its operation in 2000. Electron-positron collisions were established at centre-of-mass energies of up to $209 \mathrm{GeV}$. The maximum energy reach of LEP was thus extended by another $7 \mathrm{GeV}$ with respect to 1999 . The luminosity rate was kept high, yielding a total delivered luminosity of $233 \mathrm{pb}^{-1}$ in 2000 . High beam energy and high luminosity allowed for an extended discovery reach of LEP. In particular, the LEP experiments reported candidate events for the Higgs boson [11,12].

Performance highlights in the regime of ultra-strong damping included a maximum beam-beam parameter of 0.083 per interaction point, achieved during 1999. The beam-beam limit was not reached for operation with beam energies above $65 \mathrm{GeV}$. Using fits of the beambeam data, the beam-beam limit was inferred to be about 0.115 per interaction point (above $98 \mathrm{GeV}$ ).

The operational overhead per physics fill was reduced from $110 \mathrm{~min}$ in 1998 to $65 \mathrm{~min}$ in 2000 , maximising the time available for physics. At the same time the number of physics fills increased from 436 to 1356 per year.

On November $2^{\text {nd }}, 2000$ the LEP was shut down for good (see Figure 5) and dismantling of the accelerator started. This ended the "life" of the highest energy electron-positron collider to date and one of the largest machines that mankind has built.

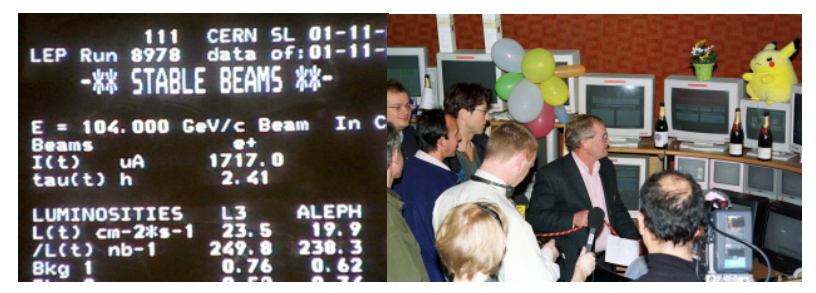

Figure 5: The public LEP page for one of the last physics fills at $104 \mathrm{GeV}$ (left) and the ceremonial dump of the last physics fill in LEP on November $2^{\text {nd }}, 2000$ (right).

\section{ACKNOWLEDGEMENTS}

The results presented are based on the scientific and technical work from many hundred people at CERN over the last 23 years. We gratefully acknowledge their work.

\section{REFERENCES}

[1] P. Janot, "What are the Priorities for LEP in its Final Year?", Chamonix 2000. CERN-SL-2000-007 DI.

[2] M. Lamont, "Strategies for Maximising Energy and Luminosity". Proceedings Chamonix 2000. CERNSL-2000-007 DI.

[3] R. Assmann, "LEP Operation and Performance with Electron-Positron Collisions at $209 \mathrm{GeV}$ '. Chamonix 2001. CERN-SL-2001-003 DI.

[4] P. Brown et al, "Ultimate Performance of the LEP RF System". These proceedings.

[5] D. Brandt et al., "Experience with a Low Emittance Optics in LEP". Proc. PAC99. CERN-SL-99-031-AP

[6] A. Beuret et al. "Proposal to Increase the LEP Energy with Horizontal Orbit Correctors". CERN-SL-2000014-MS.

[7] R. Assmann et al, "Emittance optimization with dispersion free steering at LEP". Phys. Rev. Spec. Top. Accel. Beams 3 (2000), pp.121001

[8] H. Burkhardt, "Beam Lifetime and Beam Tails in LEP”. CERN-SL-99-061-AP.

[9] R. Assmann et al, "Luminosity and Beam Measurements Used for Performance Optimisation in the LEP Collider". EPAC2000.

[10] R. Assmann and K. Cornelis, "The Beam-Beam Interaction in Storage Rings with Strong Radiation Damping”. EPAC2000.

[11] R. Barate et al, "Observation of an Excess in the Search for the Standard Model Higgs Boson at ALEPH". CERN-EP/2000-138.

[12] M. Acciarri et al, "Higgs Candidates in e+ e- Interactions at $\sqrt{s}_{\mathrm{s}}=206.6 \mathrm{GeV}$ ". CERN-EP/2000-140. 